\title{
Symmetry Reduction of the Two-Dimensional Ricci Flow Equation
}

\author{
Mehdi Nadjafikhah, ${ }^{1}$ and Mehdi Jafari ${ }^{2}$ \\ ${ }^{1}$ School of Mathematics, Iran University of Science and Technology, Narmak, Tehran 1684613114, Iran \\ ${ }^{2}$ Department of Complementary Education, Payame Noor University, P.O. Box 19395-3697, Tehran, Iran \\ Correspondence should be addressed to Mehdi Nadjafikhah; m_nadjafikhah@iust.ac.ir
}

Received 16 October 2012; Accepted 21 November 2012

Academic Editor: Salvador Hernandez

Copyright (c) 2013 M. Nadjafikhah and M. Jafari. This is an open access article distributed under the Creative Commons Attribution License, which permits unrestricted use, distribution, and reproduction in any medium, provided the original work is properly cited.

\begin{abstract}
This paper is devoted to obtain the one-dimensional group invariant solutions of the two-dimensional Ricci flow ((2D) Rf) equation. By classifying the orbits of the adjoint representation of the symmetry group on its Lie algebra, the optimal system of onedimensional subalgebras of the ((2D) Rf) equation is obtained. For each class, we will find the reduced equation by the method of similarity reduction. By solving these reduced equations, we will obtain new sets of group invariant solutions for the ((2D) Rf) equation.
\end{abstract}

\section{Introduction}

The Ricci flow was introduced by Hamilton in his seminal paper, "Three-manifolds with positive Ricci curvature" in 1982 [1]. Since then, Ricci flow has been a very useful tool for studying the special geometries which a manifold admits. Ricci flow is an evolution equation for a Riemannian metric which sometimes can be used in order to deform an arbitrary metric into a suitable metric that can specify the topology of the underlying manifold. If $(M, g(t))$ is a smooth Riemannian manifold, Ricci flow is defined by

$$
\frac{\partial}{\partial t} g(t)=-2 \text { Ric }
$$

where Ric denotes the Ricci tensor of the metric $g$. By using the concept of Ricci flow, Grisha Perelman completely proved the Poincaré conjecture around 2003 [2-4]. The Ricci flow also is used as an approximation to the renormalization group flow for the two-dimensional nonlinear $\sigma$-model, in quantum field theory; see [5] and references therein. The ricci flow equation is related to one of the models used in obtaining the quantum theory of gravity [6]. Because some difficulties appear when a quantum field theory is formulated, the studies focus on less dimensional models which are called mechanical models.
In this paper, we want to obtain new solutions of ((2D) Rf) equation by method of Lie symmetry group. As it is well known, Lie symmetry group method has an important role in the analysis of differential equations. The theory of Lie symmetry groups of differential equations was developed by Lie at the end of nineteenth century [7]. By this method, we can reduce the order of ODEs and investigate the invariant solutions. Also we can construct new solutions from known ones (for more details about the applications of Lie symmetries, see [8-10]). Lie's method led to an algorithmic approach to find special solution of differential equation by its symmetry group. These solutions are called group invariant solutions and obtained by solving the reduced system of differential equation having fewer independent variables than the original system. This fact that for some PDEs, the symmetry reductions are unobtainable by the Lie symmetry method, caused the creation of some generalizations of this method. These generalizations are called nonclassical symmetry method and was described in many references such as [11-14].

In this paper, we apply the Lie symmetry method to obtain the invariant solutions of ((2D) Rf) equation and classify them. This paper is organized as follows. In Section 2, by using the mechanical model of Ricci flow, Lie symmetries of ((2D) Rf) equation will be stated. Also we achieve some results from the structure of the Lie algebra of the Lie 
symmetry group. In Section 3, we will construct an optimal system of one-dimensional subalgebras of the ((2D) Rf) equation which is useful for classifying of the group invariant solutions. In Section 4, the reduced equation for each element of optimal system is obtained. In Section 5, we will solve the reduced equations by method of Lie symmetry group and obtain the group invariant solutions of ((2D) Rf) equation.

\section{Lie Symmetries of ((2D) Rf) Equation}

As we know, transformations which map solutions of a differential equation to other solutions are called symmetries of the equation. The procedure of finding the Lie symmetry group of a PDE was described in many studies such as $[8,9$, 15]. Before performing the Lie symmetries of Ricci flow, let us restate the mechanical model of Ricci flow that introduced by Cimpoiaus and Constantinescu [16].

The metric tensor of the space, $g_{i j}$, can be written in the conformally flat frame

$$
\begin{aligned}
d s^{2} & =g_{i j} d x^{i} d x^{j}=2 e^{\phi(x, y, t)} d x d y \\
& =\frac{1}{2} e^{\phi(X, Y, t)}\left(d X^{2}+d Y^{2}\right)
\end{aligned}
$$

using Cartesian coordinates $X$ and $Y$ or the complex variables $2 x=Y+i X, 2 y=Y-i X[6]$. According to (1), the function $\phi(X, Y, t)$ must satisfy

$$
\frac{\partial}{\partial t} e^{\phi}=\Delta \phi
$$

where $\Delta$ is Laplacian. By introducing the field

$$
u(x, y, t)=e^{\phi}
$$

Equation (3) takes the form $u_{t}=(\ln u)_{x y}$ or in the equivalent form:

$$
u^{2} u_{t}+u_{y} u_{x}-u u_{x y}=0
$$

Cimpoiaus and Constantinescu, also obtained the Lie symmetry group of this equation [16]. They proved that this equation admits a 6-parameter Lie group, $G$, with the following infinitesimal generators for its Lie algebra, $\mathfrak{g}$ :

$$
\begin{gathered}
X_{1}=\partial_{x}, \quad X_{2}=\partial_{y}, \quad X_{3}=\partial_{t}, \\
X_{4}=t \partial_{t}+u \partial_{u}, \quad X_{5}=x \partial_{x}-u \partial_{u}, \quad X_{6}=y \partial_{y}-u \partial_{u} .
\end{gathered}
$$

The commutator table of Lie algebra for $\mathfrak{g}$ is given in Table 1 , where the entry in the $i$ th row and $j$ th column is $\left[X_{i}, X_{j}\right]=$ $X_{i} X_{j}-X_{j} X_{i}, i, j=1, \ldots, 6$.
TABLE 1: The commutator table of $\mathfrak{g}$.

\begin{tabular}{ccccccc}
\hline$[]$, & $X_{1}$ & $X_{2}$ & $X_{3}$ & $X_{4}$ & $X_{5}$ & $X_{6}$ \\
\hline$X_{1}$ & 0 & 0 & 0 & 0 & $X_{1}$ & 0 \\
$X_{2}$ & 0 & 0 & 0 & 0 & 0 & $X_{2}$ \\
$X_{3}$ & 0 & 0 & 0 & $X_{3}$ & 0 & 0 \\
$X_{4}$ & 0 & 0 & $-X_{3}$ & 0 & 0 & 0 \\
$X_{5}$ & $-X_{1}$ & 0 & 0 & 0 & 0 & 0 \\
$X_{6}$ & 0 & $-X_{2}$ & 0 & 0 & 0 & 0 \\
\hline
\end{tabular}

Exponentiating the infinitesimal symmetries (6), we obtain the one-parameter groups $g_{k}(s)$ generated by $X_{k}, k=$ $1, \ldots, 6$ as follows:

$$
\begin{aligned}
& g_{1}(s):(x, y, t, u) \longmapsto(x+s, y, t, u), \\
& g_{2}(s):(x, y, t, u) \longmapsto(x, y+s, t, u), \\
& g_{3}(s):(x, y, t, u) \longmapsto(x, y, t+s, u), \\
& g_{4}(s):(x, y, t, u) \longmapsto\left(x, y, t e^{s}, u e^{s}\right), \\
& g_{5}(s):(x, y, t, u) \longmapsto\left(x e^{s}, y, t, u e^{-s}\right), \\
& g_{6}(s):(x, y, t, u) \longmapsto\left(x, y e^{s}, t, u e^{-s}\right) .
\end{aligned}
$$

Consequently, we can state the following theorem.

Theorem 1. If $f=f(x, y, t)$ is a solution of (5), so are functions

$$
\begin{aligned}
& g_{1}(s) \cdot f=f(x-s, y, t), \\
& g_{2}(s) \cdot f=f(x, y-s, t), \\
& g_{3}(s) \cdot f=f(x, y, t-s), \\
& g_{4}(s) \cdot f=f\left(x, y, t e^{-s}\right) e^{s}, \\
& g_{5}(s) \cdot f=f\left(x e^{-s}, y, t\right) e^{-s}, \\
& g_{6}(s) \cdot f=f\left(x, y e^{-s}, t\right) e^{-s} .
\end{aligned}
$$

\section{One-Dimensional Optimal System of Subalgebras for the ((2D) Rf) Equation}

In this section, we obtain the one-dimensional optimal system of ((2D) Rf) equation by using symmetry group. Since every linear combination of infinitesimal symmetries is an infinitesimal symmetry, there is an infinite number of onedimensional subgroups for $G$. Therefore, it is important to determine which subgroups give different types of solutions. For this, we must find invariant solutions which cannot be transformed to each other by symmetry transformations in the full symmetry group. This led to the concept of an optimal system of subalgebra. For one-dimensional subalgebras, this classification problem is the same as the problem of classifying the orbits of the adjoint representation [8]. Optimal set of subalgebras is obtained by selecting only one representative from each class of equivalent subalgebras. The problem of classifying the orbits is solved by taking a general element 
TABLE 2: The adjoint representation table of the infinitesimal generators $X_{i}$.

\begin{tabular}{ccccccc}
\hline Ad & $X_{1}$ & $X_{2}$ & $X_{3}$ & $X_{4}$ & $X_{5}$ & $X_{6}$ \\
\hline$X_{1}$ & $X_{1}$ & $X_{2}$ & $X_{3}$ & $X_{4}$ & $X_{5}-s X_{1}$ & $X_{6}$ \\
$X_{2}$ & $X_{1}$ & $X_{2}$ & $X_{3}$ & $X_{4}$ & $X_{5}$ & $X_{6}-s X_{2}$ \\
$X_{3}$ & $X_{1}$ & $X_{2}$ & $X_{3}$ & $X_{4}-s X_{3}$ & $X_{5}$ & $X_{6}$ \\
$X_{4}$ & $X_{1}$ & $X_{2}$ & $e^{s} X_{3}$ & $X_{4}$ & $X_{5}$ & $X_{6}$ \\
$X_{5}$ & $e^{s} X_{1}$ & $X_{2}$ & $X_{3}$ & $X_{4}$ & $X_{5}$ & $X_{6}$ \\
$X_{6}$ & $X_{1}$ & $e^{s} X_{2}$ & $X_{3}$ & $X_{4}$ & $X_{5}$ & $X_{6}$ \\
\hline
\end{tabular}

in the Lie algebra and simplifying it as much as possible by imposing various adjoint transformation on it $[15,17]$. Adjoint representation of each $X_{i}, i=1, \ldots, 6$ is defined by Lie series

$$
\begin{aligned}
\operatorname{Ad}\left(\exp \left(s \cdot X_{i}\right) \cdot X_{j}\right)= & X_{j}-s \cdot\left[X_{i}, X_{j}\right] \\
& +\frac{s^{2}}{2} \cdot\left[X_{i},\left[X_{i}, X_{j}\right]\right]-\cdots,
\end{aligned}
$$

where $s$ is a parameter and $\left[X_{i}, X_{j}\right]$ is the commutator of the Lie algebra for $i, j=1, \ldots, 6[8]$. It is important to note that following the convention of [8], we used the right invariant vector fields to define the Lie algebra in this paper. As a consequence a minus sign is present in Lie series.

Taking into account the table of commutator, we can compute all the adjoint representations corresponding to the Lie group of the $((2 \mathrm{D}) \mathrm{Rf})$ equation. They are presented in Table 2 . Note that, the $(i, j)$ entry indicate $\operatorname{Ad}\left(\exp \left(s \cdot X_{i}\right) \cdot X_{j}\right)$.

Now we can state the following theorem.

Theorem 2. A one-dimensional optimal system for Lie algebra of ((2D) $R f)$ equation is given by
(1) $X_{1}+a X_{2}+b X_{3}$,
(2) $X_{1} \pm X_{2}+c X_{4}$,
(3) $X_{1} \pm X_{3}+c X_{6}$,
(4) $X_{1}+c X_{4}+d X_{6}$
(5) $X_{2} \pm X_{3}+c X_{5}$,
(6) $X_{2}+c X_{4}+d X_{5}$,
(7) $X_{3}+c X_{5}+d X_{6}$,
(8) $X_{4}+c X_{5}+d X_{6}$,

where $a, b, c, d \in \mathbb{R}$ and $a \neq 0, b \neq 0$.
Proof. Let $F_{i}^{s}: \mathfrak{g} \rightarrow \mathfrak{g}$ be the adjoint transformation defined by $X \mapsto \operatorname{Ad}\left(\exp \left(s X_{i}\right) \cdot X\right)$, for $i=1, \ldots, 6$. The matrix of $F_{i}^{s}$, $i=1, \ldots, 6$, with respect to basis $\left\{X_{1}, \ldots, X_{6}\right\}$ is

$$
M_{1}^{s}=\left[\begin{array}{cccccc}
1 & 0 & 0 & 0 & 0 & 0 \\
0 & 1 & 0 & 0 & 0 & 0 \\
0 & 0 & 1 & 0 & 0 & 0 \\
0 & 0 & 0 & 1 & 0 & 0 \\
-s & 0 & 0 & 0 & 1 & 0 \\
0 & 0 & 0 & 0 & 0 & 1
\end{array}\right], \quad M_{2}^{s}=\left[\begin{array}{cccccc}
1 & 0 & 0 & 0 & 0 & 0 \\
0 & 1 & 0 & 0 & 0 & 0 \\
0 & 0 & 1 & 0 & 0 & 0 \\
0 & 0 & 0 & 1 & 0 & 0 \\
0 & 0 & 0 & 0 & 1 & 0 \\
0 & -s & 0 & 0 & 0 & 1
\end{array}\right],
$$

$$
\begin{array}{rlrl}
M_{3}^{s} & =\left[\begin{array}{cccccc}
1 & 0 & 0 & 0 & 0 & 0 \\
0 & 1 & 0 & 0 & 0 & 0 \\
0 & 0 & 1 & 0 & 0 & 0 \\
0 & 0 & -s & 1 & 0 & 0 \\
0 & 0 & 0 & 0 & 1 & 0 \\
0 & 0 & 0 & 0 & 0 & 1
\end{array}\right], & M_{4}^{s}=\left[\begin{array}{llllll}
1 & 0 & 0 & 0 & 0 & 0 \\
0 & 1 & 0 & 0 & 0 & 0 \\
0 & 0 & e^{s} & 0 & 0 & 0 \\
0 & 0 & 0 & 1 & 0 & 0 \\
0 & 0 & 0 & 0 & 1 & 0 \\
0 & 0 & 0 & 0 & 0 & 1
\end{array}\right], \\
M_{5}^{s} & =\left[\begin{array}{cccccc}
e^{s} & 0 & 0 & 0 & 0 & 0 \\
0 & 1 & 0 & 0 & 0 & 0 \\
0 & 0 & 1 & 0 & 0 & 0 \\
0 & 0 & 0 & 1 & 0 & 0 \\
0 & 0 & 0 & 0 & 1 & 0 \\
0 & 0 & 0 & 0 & 0 & 1
\end{array}\right], & M_{6}^{s} & =\left[\begin{array}{llllll}
1 & 0 & 0 & 0 & 0 & 0 \\
0 & e^{s} & 0 & 0 & 0 & 0 \\
0 & 0 & 1 & 0 & 0 & 0 \\
0 & 0 & 0 & 1 & 0 & 0 \\
0 & 0 & 0 & 0 & 1 & 0 \\
0 & 0 & 0 & 0 & 0 & 1
\end{array}\right],
\end{array}
$$

respectively. If $X=\sum_{i=1}^{6} a_{i} X_{i}$, then we have

$$
\begin{aligned}
& F_{6}^{s_{6}} \circ F_{5}^{s_{5}} \circ \cdots \circ F_{1}^{s_{1}}: X \\
& \qquad e^{s_{5}} a_{1} X_{1}+e^{s_{6}} a_{2} X_{2}+e^{s_{4}} a_{3} X_{3}+\left(a_{4}-e^{s_{4}} s_{3} a_{3}\right) X_{4} \\
& \quad+\left(a_{5}-e^{s_{5}} s_{1} a_{1}\right) X_{5}+\left(a_{6}-e^{s_{6}} s_{2} a_{2}\right) X_{6} .
\end{aligned}
$$

Now, we try to vanish the coefficients of $X$ by acting the adjoint representations $M_{i}^{s_{i}}$ on $X$, and choosing suitable parameters $s_{i}$ in each step. Therefore, we can simplify $X$ as follows.

If $a_{1} \neq 0, a_{2} \neq 0$ and $a_{3} \neq 0$, then we can make the coefficients of $X_{5}, X_{6}$, and $X_{4}$ vanish by $F_{1}^{S_{1}}, F_{2}^{S_{2}}$, and $F_{3}^{S_{3}}$; by setting $s_{1}=a_{5} / a_{1}, s_{2}=a_{6} / a_{2}$ and $s_{3}=a_{4} / a_{3}$, respectively. Scaling $X$ if necessary, we can assume that $a_{1}=1$. So $X$ is reduced to the case (1).

If $a_{1} \neq 0, a_{2} \neq 0$ and $a_{3}=0$, then we can make the coefficients of $X_{5}$ and $X_{6}$ vanish by $F_{1}^{S_{1}}$ and $F_{2}^{S_{2}}$; by setting $s_{1}=a_{5} / a_{1}$ and $s_{2}=a_{6} / a_{2}$, respectively. Also we can make the coefficient of $X_{2}, \pm 1$ by $F_{6}^{S_{6}}$; by setting $s_{6}=-\ln \left|a_{2}\right|$. Scaling $X$ if necessary, we can assume that $a_{1}=1$. So $X$ is reduced to the case (2).

If $a_{1} \neq 0, a_{3} \neq 0$ and $a_{2}=0$, then we can make the coefficients of $X_{5}$ and $X_{4}$ vanish by $F_{1}^{S_{1}}$ and $F_{3}^{S_{3}}$; by setting $s_{1}=a_{5} / a_{1}$ and $s_{3}=a_{4} / a_{3}$, respectively. Also we can make the coefficient of $X_{3}, \pm 1$ by $F_{4}^{S_{4}}$; by setting $s_{4}=-\ln \left|a_{3}\right|$. Scaling $X$ if necessary, we can assume that $a_{1}=1$. So $X$ is reduced to the case (3).

If $a_{1} \neq 0$ and $a_{2}=a_{3}=0$, then we can make the coefficient of $X_{5}$ vanish by $F_{1}^{S_{1}}$; by setting $s_{3}=a_{5} / a_{1}$. 
TABLE 3: Lie invariants, similarity solutions, and reduced equations.

\begin{tabular}{lllll}
\hline$i$ & $\mathfrak{h}_{i}$ & $\left\{z_{i}, w_{i}, v_{i}\right\}$ & $u_{i}$ & Similarity reduced equations \\
\hline 1 & $X_{1}+X_{6}$ & $\left\{y e^{-x}, t, u y\right\}$ & $\frac{f(z, w)}{y}$ & $f^{2} f_{w}-z^{2} f_{z}^{2}+z f f_{z}+z^{2} f f_{z z}=0$ \\
2 & $X_{2}+X_{4}$ & $\left\{x, t e^{-y}, u e^{-y}\right\}$ & $f(z, w) e^{y}$ & $f^{2} f_{w}-w f_{z} f_{w}+w f f_{z w}=0$ \\
3 & $X_{3}+X_{5}+d X_{6}$ & $\left\{\frac{y}{x^{d}}, \ln \frac{e^{t}}{x}, u x^{d+1}\right\}$ & $\frac{f(z, w)}{x^{d+1}}$ & $f_{w}\left(f^{2}-f_{z}\right)-d z f_{z}^{2}+f\left(d f_{z}+d z f_{z z}+f_{z w}\right)=0$ \\
4 & $X_{2}+X_{3}+X_{5}$ & $\left\{\ln \frac{e^{y}}{x}, \ln \frac{e^{t}}{x}, u x\right\}$ & $\frac{f(z, w)}{x}$ & $f^{2} f_{w}-f_{z}^{2}-f_{w} f_{z}+f f_{z z}+f f_{z w}=0$ \\
5 & $X_{2}+X_{5}$ & $\left\{\ln \frac{e^{y}}{x}, t, u x\right\}$ & $\frac{f(z, w)}{x}$ & $f^{2} f_{w}-f_{z}^{2}+f f_{z z}=0$ \\
6 & $X_{3}+X_{6}$ & $\{x, t-\ln y, u y\}$ & $\frac{f(z, w)}{y}$ & $f^{2} f_{w}-f_{z} f_{w}+f f_{z w}=0$ \\
7 & $X_{1}+X_{2}$ & $\{y-x, t, u\}$ & $f(z, w)$ & $f^{2} f_{w}-f_{z}^{2}+f f_{z z}=0$ \\
8 & $X_{2}+X_{3}$ & $\{x, t-y, u\}$ & $f(z, w)$ & $f^{2} f_{w}-f_{z} f_{w}+f f_{z w}=0$ \\
\hline
\end{tabular}

Scaling $X$ if necessary, we can assume that $a_{1}=1$. So $X$ is reduced to the case (4).

If $a_{2} \neq 0, a_{3} \neq 0$ and $a_{1}=0$, then we can make the coefficients of $X_{6}$ and $X_{4}$ vanish by $F_{2}^{S_{2}}$ and $F_{3}^{S_{3}}$; by setting $s_{2}=a_{6} / a_{2}$ and $s_{3}=a_{4} / a_{3}$, respectively. Also we can make the coefficient of $X_{3}, \pm 1$ by $F_{4}^{S_{4}}$; by setting $s_{4}=-\ln \left|a_{3}\right|$. Scaling $X$ if necessary, we can assume that $a_{2}=1$. So $X$ is reduced to the case (5).

If $a_{2} \neq 0$ and $a_{1}=a_{3}=0$, then we can make the coefficient of $X_{6}$ vanish by $F_{2}^{S_{2}}$; by setting $s_{2}=a_{6} / a_{2}$. Scaling $X$ if necessary, we can assume that $a_{2}=1$. So $X$ is reduced to the case (6).

If $a_{1}=a_{2}=0$ and $a_{3} \neq 0$, then we can make the coefficient of $X_{4}$ vanish by $F_{3}^{S_{3}}$; by setting $s_{3}=a_{4} / a_{3}$. Scaling $X$ if necessary, we can assume that $a_{3}=1$. So $X$ is reduced to the case (7).

If $a_{1}=a_{2}=a_{3}=0$, then $X$ is reduced to the case (8).

There is not any more possible case for investigating and the proof is complete.

\section{Similarity Reduction of ((2D) Rf) Equation}

In this section, the two-dimensional Ricci flow equation will be reduced by expressing it in the new coordinates. The ((2D) Rf) equation is expressed in the coordinates $(x, y, t, u)$, we must search for this equation's form in the suitable coordinates for reducing it. These new coordinates will be obtained by looking for independent invariants $(z, w, f)$ corresponding to the generators of the symmetry group. Hence, by using the new coordinates and applying the chain rule, we obtain the reduced equation. We express this procedure for one of the infinitesimal generators in the optimal system (10) and list the result for some other cases.

For example, consider the case (4) in Theorem 2 when $c=0$ and $d=1$; therefore, we have $X:=X_{1}+X_{6}$. For determining independent invariants $I$, we ought to solve the PDEs $X(I)=0$, that is

$$
\begin{aligned}
\left(X_{1}+X_{6}\right) I & =\left(\partial_{x}+y \partial_{y}-u \partial_{u}\right) I \\
& =\frac{\partial I}{\partial x}+y \frac{\partial I}{\partial y}+0 \frac{\partial I}{\partial t}-u \frac{\partial I}{\partial u}=0 .
\end{aligned}
$$

For solving this PDE, the following associated characteristic ODE must be solved:

$$
\frac{d x}{1}=\frac{d y}{y}=\frac{d t}{0}=\frac{d u}{-u}
$$

Hence, three functionally independent invariants $z=y e^{-x}$, $w=t$, and $f=u y$ are obtained. If we treat $f$ as a function of $z$ and $w$, we can compute formulae for the derivatives of $u$ with respect to $x, y$, and $t$ in terms of $z, w, f$ and the derivatives of $f$ with respect to $z$ and $w$. By using the chain rule and the fact that $u=f(z, w) y^{-1}$, we have

$$
\begin{gathered}
u_{t}=\left(f_{z} z_{t}+f_{w} w_{t}\right) y^{-1}=f_{w} y^{-1}, \\
u_{x}=-f_{z} e^{-x} \\
u_{y}=f_{z} e^{-x} y^{-1}-f y^{-2} \\
u_{x y}=-e^{-2 x} f_{z z} .
\end{gathered}
$$

After substituting the above relations into (5), we obtain

$$
\begin{aligned}
& u^{2} u_{t}+u_{y} u_{x}-u u_{x y} \\
& =y^{-3}\left(f^{2} f_{w}-f_{z}^{2} z^{2}+f f_{z} z+f f_{z z} z^{2}\right)=0 .
\end{aligned}
$$

So the reduced equation is

$$
f^{2} f_{w}-z^{2} f_{z}^{2}+z f f_{z}+z^{2} f f_{z z}=0 .
$$

This equation has two independent variables $z$ and $w$ and one dependent variable $f$. In a similar way, we can compute all of the similarity reduction equations corresponding to the infinitesimal symmetries that mentioned in Theorem 2 . Some of them are listed in Table 3. 
TABLE 4: ODEs obtained from the reduced equations of Table 3.

\begin{tabular}{|c|c|c|c|c|}
\hline \multicolumn{2}{|r|}{$i$ Symmetry group generators } & Optimal system & Invariants $\{s, g\}$ & Reduced equation \\
\hline 1 & $\begin{array}{l}V_{1}=\frac{1}{2} z \ln z \partial_{z}+w \partial_{w} \\
V_{2}=\partial_{w} \\
V_{3}=-\frac{1}{2} z \ln z \partial_{z}+f \partial_{f} \\
V_{4}=z \partial_{z}\end{array}$ & $\begin{array}{l}\mathscr{A}_{1}^{1}: V_{2} \\
\mathscr{A}_{1}^{2}: V_{3} \\
\mathscr{A}_{1}^{3}: V_{1}+V_{3} \\
\mathscr{A}_{1}^{4}: V_{2}+V_{4}\end{array}$ & $\begin{array}{l}\{z, f\} \\
\left\{w, f(\ln z)^{2}\right\} \\
\left\{z, \frac{f}{w}\right\} \\
\{w-\ln z, f\}\end{array}$ & $\begin{array}{l}s g^{\prime 2}-g g^{\prime}-s g g^{\prime \prime}=0 \\
g^{2}\left(g^{\prime}+2\right)=0 \\
g^{3}-s^{2} g^{\prime 2}+s g g^{\prime}+s^{2} g g^{\prime \prime}=0 \\
g^{2} g^{\prime}-g^{\prime 2}+g g^{\prime \prime}=0\end{array}$ \\
\hline 2 & $\begin{array}{l}V_{1}=w \partial_{w}+f \partial_{f} \\
V_{2}=z \partial_{z}-f \partial_{f} \\
V_{3}=\partial_{z}\end{array}$ & $\begin{array}{l}\mathscr{A}_{2}^{1}: V_{2} \\
\mathscr{A}_{2}^{2}: V_{3}\end{array}$ & $\begin{array}{l}\{w, f z\} \\
\{w, f\}\end{array}$ & $\begin{array}{l}g^{2} g^{\prime}=0 \\
g^{2} g^{\prime}=0\end{array}$ \\
\hline 3 & $\begin{array}{l}V_{1}=z \ln z \partial_{z}+w \partial_{w}-f(1+\ln z) \partial_{f} \\
V_{2}=\partial_{w} \\
V_{3}=z \partial_{z}-f \partial_{f}\end{array}$ & $\begin{array}{l}\mathscr{A}_{3}^{1}: V_{1} \\
\mathscr{A}_{3}^{2}: V_{2} \\
\mathscr{A}_{3}^{3}: V_{3}\end{array}$ & $\begin{array}{l}\left\{\frac{w}{\ln z}, f z \ln z\right\} \\
\{z, f\} \\
\{w, f z\}\end{array}$ & $\begin{array}{l}g g^{\prime}(g+2 d s-1)+d g^{2}+s(d s-1)\left(g g^{\prime \prime}-g^{\prime 2}\right)=0 \\
s g^{\prime 2}-g g^{\prime}-s g g^{\prime \prime}=0 \\
g^{2} g^{\prime}=0\end{array}$ \\
\hline 4 & $\begin{array}{l}V_{1}=z \partial_{z}+w \partial_{w}-f \partial_{f} \\
V_{2}=\partial_{w} \\
V_{3}=\partial_{z}\end{array}$ & $\begin{array}{l}\mathscr{A}_{4}^{1}: V_{1} \\
\mathscr{A}_{4}^{2}: V_{2} \\
\mathscr{A}_{4}^{3}: V_{2}-V_{3}\end{array}$ & $\begin{array}{l}\left\{\frac{w}{z}, f z\right\} \\
\{z, f\} \\
\{z+w, f\}\end{array}$ & $\begin{array}{l}g g^{\prime}(1-g-2 s)-g^{2}+s(s-1)\left(g^{\prime 2}-g g^{\prime \prime}\right)=0 \\
-g^{\prime 2}+g g^{\prime \prime}=0 \\
g^{2} g^{\prime}-2 g^{\prime 2}+2 s g g^{\prime \prime}=0\end{array}$ \\
\hline 5 & $\begin{array}{l}V_{1}=\frac{z}{2} \partial_{z}+w \partial_{w} \\
V_{2}=\partial_{w} \\
V_{3}=-\frac{z}{2} \partial_{z}+f \partial_{f} \\
V_{4}=\partial_{z}\end{array}$ & $\begin{array}{l}\mathscr{A}_{5}^{1}: V_{3} \\
\mathscr{A}_{5}^{2}: V_{1}+V_{3} \\
\mathscr{A}_{5}^{3}: V_{2}+V_{4}\end{array}$ & $\begin{array}{l}\left\{w, f z^{2}\right\} \\
\left\{z, \frac{f}{w}\right\} \\
\{w-z, f\}\end{array}$ & $\begin{array}{l}g^{\prime}+2=0 \\
g^{3}-g^{\prime 2}+g g^{\prime \prime}=0 \\
g^{2} g^{\prime}-g^{\prime 2}+g g^{\prime \prime}=0\end{array}$ \\
\hline 6 & $\begin{array}{l}V_{1}=w \partial_{w} \\
V_{2}=\partial_{w} \\
V_{3}=z \partial_{z}-f \partial_{f} \\
V_{4}=\partial_{z}\end{array}$ & $\begin{array}{l}\mathscr{A}_{6}^{1}: V_{4} \\
\mathscr{A}_{6}^{2}: V_{2}+V_{3}\end{array}$ & $\begin{array}{l}\{w, f\} \\
\{w-\ln z, z f\}\end{array}$ & $\begin{array}{l}g^{\prime}=0 \\
g^{2} g^{\prime}+g^{\prime 2}-g g^{\prime \prime}=0\end{array}$ \\
\hline 7 & $\begin{array}{l}V_{1}=\frac{z}{2} \partial_{z}+w \partial_{w} \\
V_{2}=\partial_{w} \\
V_{3}=-\frac{z}{2} \partial_{z}+f \partial_{f} \\
V_{4}=\partial_{z}\end{array}$ & $\begin{array}{l}\mathscr{A}_{7}^{1}: V_{2} \\
\mathscr{A}_{7}^{2}: V_{3} \\
\mathscr{A}_{7}^{3}: V_{2}+V_{4} \\
\mathscr{A}_{7}^{4}: V_{1}-V_{3}\end{array}$ & $\begin{array}{l}\{z, f\} \\
\left\{w, f z^{2}\right\} \\
\{w-z, f\} \\
\left\{\frac{w}{z}, f z\right\}\end{array}$ & $\begin{array}{l}g^{\prime 2}-g g^{\prime \prime}=0 \\
g^{2}\left(g^{\prime}+2\right)=0 \\
g^{2} g^{\prime}-g^{\prime 2}+g g^{\prime \prime}=0 \\
g^{2} g^{\prime}-s^{2} g^{\prime 2}+2 s g g^{\prime}+g^{2}+s^{2} g g^{\prime \prime}=0\end{array}$ \\
\hline 8 & $\begin{array}{l}V_{1}=w \partial_{w} \\
V_{3}=z \partial_{z}-f \partial_{f} \\
V_{2}=\partial_{w} \\
V_{4}=\partial_{z}\end{array}$ & $\begin{array}{l}\mathscr{A}_{8}^{1}: V_{2}+V_{4} \\
\mathscr{A}_{8}^{2}: V_{2}+V_{3}\end{array}$ & $\begin{array}{l}\{w-z, f\} \\
\{w-\ln z, z f\}\end{array}$ & $\begin{array}{l}g^{2} g^{\prime}+g^{\prime 2}-g g^{\prime \prime}=0 \\
g^{2} g^{\prime}+g^{\prime 2}-g g^{\prime \prime}=0\end{array}$ \\
\hline
\end{tabular}

\section{Group Invariant Solutions of ((2D) Rf) Equation}

In this section, we reduce the equations obtained in last section to ODEs and solve them.

For example, (17) admits a 4-parameter family of Lie operators with following infinitesimal generators:

$$
\begin{gathered}
V_{1}=\frac{1}{2} z \ln z \partial_{z}+w \partial_{w}, \\
V_{2}=\partial_{w}, \\
V_{3}=-\frac{1}{2} z \ln z \partial_{z}+f \partial_{f}, \\
V_{4}=z \partial_{z} .
\end{gathered}
$$

The invariants associated to the infinitesimal generator $V_{2}$, are $s=z$ and $g=f$. By substituting these invariants into (17) and using chain rule, the reduced equation is obtained as follows:

$$
s g^{\prime 2}-g g^{\prime}-s g g^{\prime \prime}=0
$$

the solution of this equation is $g(s)=c_{2} s^{c_{1}}=c_{2} z^{c_{1}}$, where $c_{1}$ and $c_{2}$ are arbitrary constants. therefore we have $f(z)=$ $c_{2} z^{c_{1}}=c_{2}\left(y e^{-x}\right)^{c_{1}}=c_{2} y^{c_{1}} e^{-c_{1} x}$. So $u=f y^{-1}=c_{2} y^{c_{1}-1} e^{-c_{1} x}$ is a solution of (5).

By similar arguments, we can obtain other invariant solutions of (17). Also by reducing other equations in Table 3 , we can find other solutions of ((2D) Rf) equation. Some of the similarity reduced equations are listed in Table 4. 
TABLE 5: Group invariant solutions of the ((2D) Rf) equation.

\begin{tabular}{ll}
\hline $\mathscr{A}_{j}^{i}$ & Invariant solution \\
\hline $\mathscr{A}_{1}^{2}$ & $-2 s+c_{1}$ \\
$\mathscr{A}_{1}^{3}$ & $\frac{1}{2 c_{1}^{2}}\left(1-\tanh \left(\frac{\ln s-c_{2}}{2 c_{1}}\right)^{2}\right)$ \\
$\mathscr{A}_{2}^{1}$ & $c_{1}$ \\
$\mathscr{A}_{3}^{2}$ & $c_{2} s^{c_{1}}$ \\
$\mathscr{A}_{3}^{1}$ & $\frac{c_{1}\left(1+c_{1}\right) s^{c_{1}}}{-s^{c_{1}}\left(1+c_{1}-d s\right)+d c_{1} c_{2}\left(1+c_{1}\right)(d s-1)^{c_{1}+1}}$ \\
$\mathscr{A}_{4}^{3}$ & $\frac{2 c_{1} e^{c_{1}\left(s+c_{2}\right)}}{-1+e^{c_{1}\left(s+c_{2}\right)}}$ \\
$\mathscr{A}_{5}^{2}$ & $\frac{1}{2 c_{1}^{2}}\left(1-\tanh \left(\frac{s+c_{2}}{2 c_{1}}\right)^{2}\right)$ \\
$\mathscr{A}_{7}^{1}$ & $c_{2} e^{c_{1} s}$ \\
$\mathscr{A}_{7}^{4}$ & $\frac{-c_{1}^{2} e^{c_{1} / s}}{e^{c_{1} / s}\left(c_{1}-s\right)-s c_{1}^{2} c_{2}}$ \\
$\mathscr{A}_{8}^{1}$ & $\frac{c_{1} e^{c_{1}\left(s+c_{2}\right)}}{1-e^{c_{1}\left(s+c_{2}\right)}}$ \\
\hline
\end{tabular}

In Table 5, we obtain the invariant solutions of ((2D) Rf) equation corresponding to some of the similarity-reduced equations.

\section{Conclusion}

In this paper, by using the adjoint representation of the symmetry group on its Lie algebra, we have constructed an optimal system of one-dimensional subalgebras for a wellknown partial differential equation in mathematical physics called: two-dimensional Ricci flow equation. Moreover, we have obtained the similarity-reduced equations for each element of optimal system as well as some group invariant solutions of two-dimensional Ricci flow equation.

\section{References}

[1] R. S. Hamilton, "Three-manifolds with positive Ricci curvature," Journal of Differential Geometry, vol. 17, no. 2, pp. 255-306, 1982.

[2] G. Perelman, "Finite extinction time for the solutions to the Ricci flow on certain three-manifolds," http://arxiv.org/ abs/math/0307245.

[3] G. Perelman, "Ricci flow with surgery on three-manifolds," http://arxiv.org/abs/math.DG/0303109.

[4] G. Perelman, "The entropy formula for the Ricci flow and its geometric applications," http://arxiv.org/abs/math.DG/ 0211159 .

[5] K. Gawedzki, "Lectures on conformal field theory," in Quantum Fields and Strings: A Course for Mathematicians, pp. 727-805, American Mathematical Society, Princeton, NJ, USA, 1996-97.

[6] I. Bakas, "Ricci flows and infinite dimensional algebras," Fortschritte der Physik, vol. 52, no. 6-7, pp. 464-471, 2004.

[7] S. Lie, "On integration of a class of linear partial differential equations by means of definite integrals," Archive for Mathematical Logic, vol. 6, pp. 328-368, 1881, translation by Ibragimov, N. H.
[8] P. J. Olver, Applications of Lie Groups to Differential Equations, Springer, New York, NY, USA, 1986.

[9] G. W. Bluman and J. D. Cole, Similarity Methods for Differential Equations, vol. 13 of Applied Mathematical Sciences, Springer, New York, NY, USA, 1974.

[10] G. W. Bluman and S. Kumei, Symmetries and Differential Equations, Springer, New York, NY, USA, 1989.

[11] P. J. Olver and P. Rosenau, "Group-invariant solutions of differential equations," SIAM Journal on Applied Mathematics, vol. 47, no. 2, pp. 263-278, 1987.

[12] R. Z. Zhdanov, I. M. Tsyfra, and R. O. Popovych, "A precise definition of reduction of partial differential equations," Journal of Mathematical Analysis and Applications, vol. 238, no. 1, pp. 101-123, 1999.

[13] G. Cicogna, "A discussion on the different notions of symmetry of differential equations," Proceedings of Institute of Mathematics of NAS of Ukraine, vol. 50, pp. 77-84, 2004.

[14] G. W. Bluman and J. D. Cole, "The general similarity solutions of the heat equation," Journal of Mathematics and Mechanics, vol. 18, pp. 1025-1042, 1969.

[15] L. V. Ovsiannikov, Group Analysis of Differential Equations, Academic Press, New York, NY, USA, 1982.

[16] R. Cimpoiasu and R. Constantinescu, "Symmetries and invariants for the 2D-Ricci flow model," Journal of Nonlinear Mathematical Physics, vol. 13, no. 2, pp. 285-292, 2006.

[17] M. Nadjafikhah, "Lie symmetries of inviscid burgers' equation," Advances in Applied Clifford Algebras, vol. 19, no. 1, pp. 101-112, 2009. 


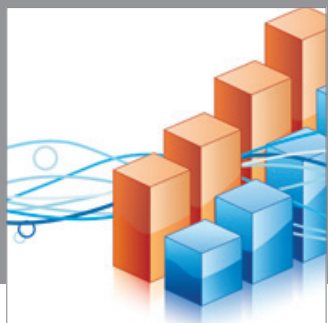

Advances in

Operations Research

mansans

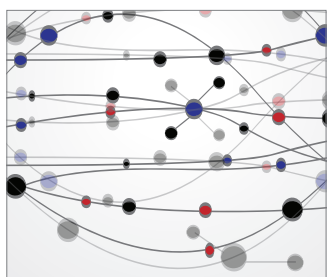

The Scientific World Journal
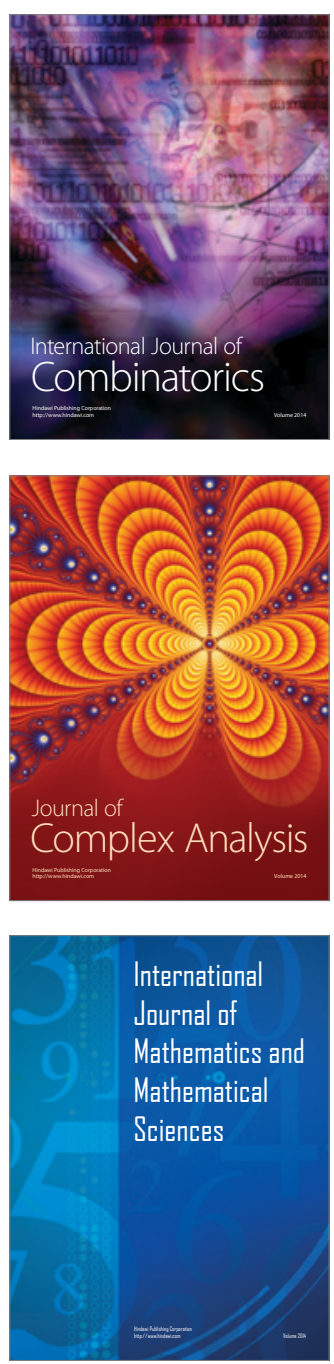
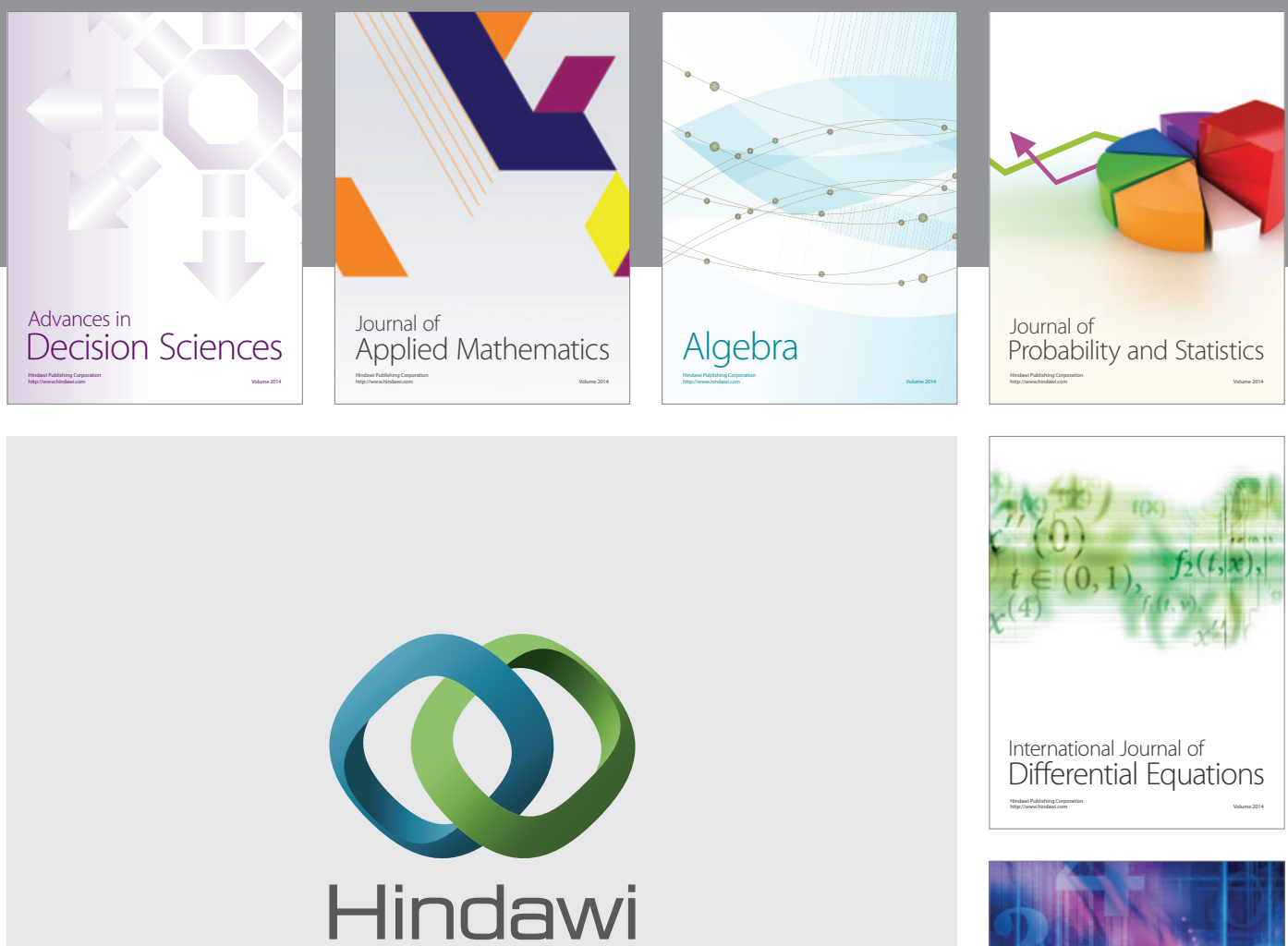

Submit your manuscripts at http://www.hindawi.com
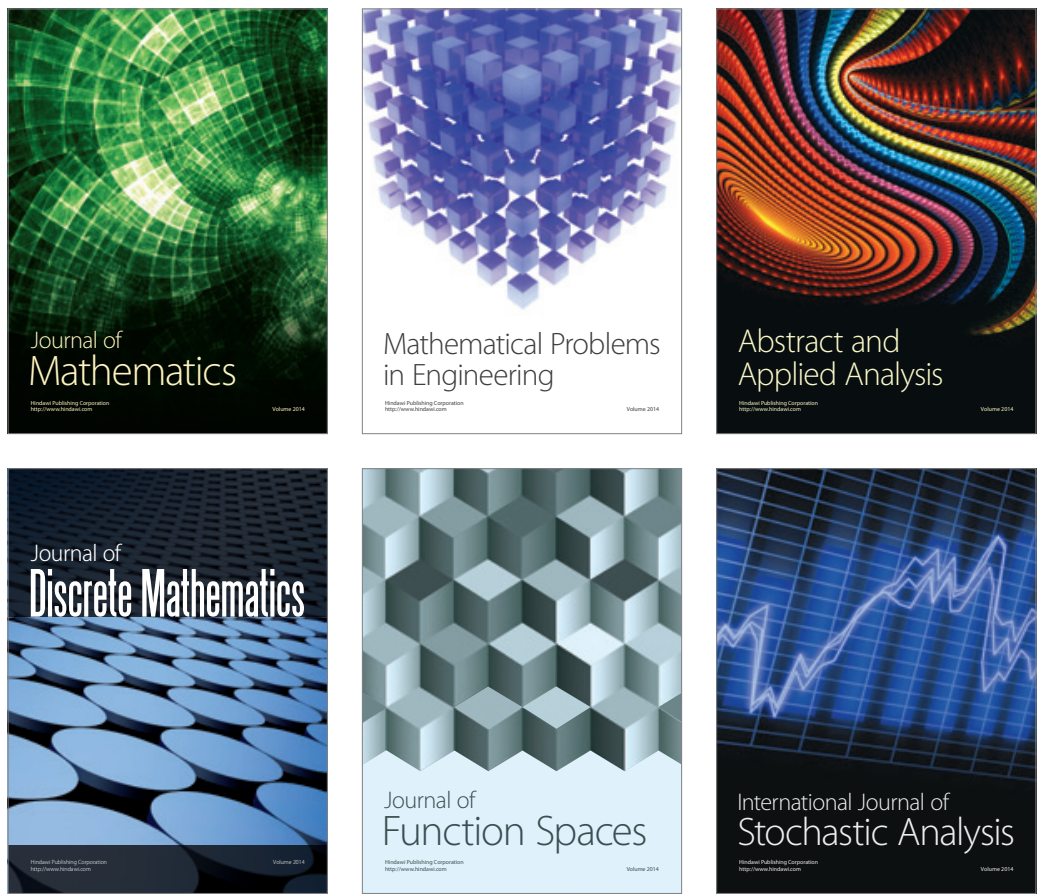

Journal of

Function Spaces

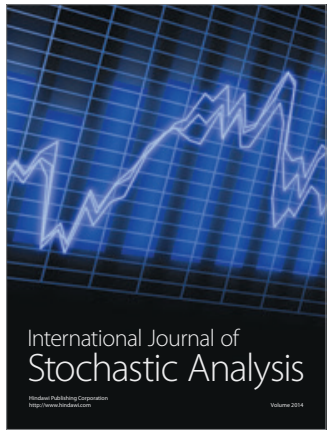

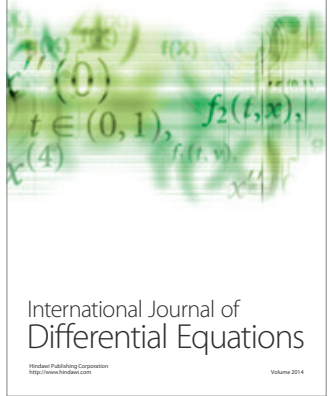
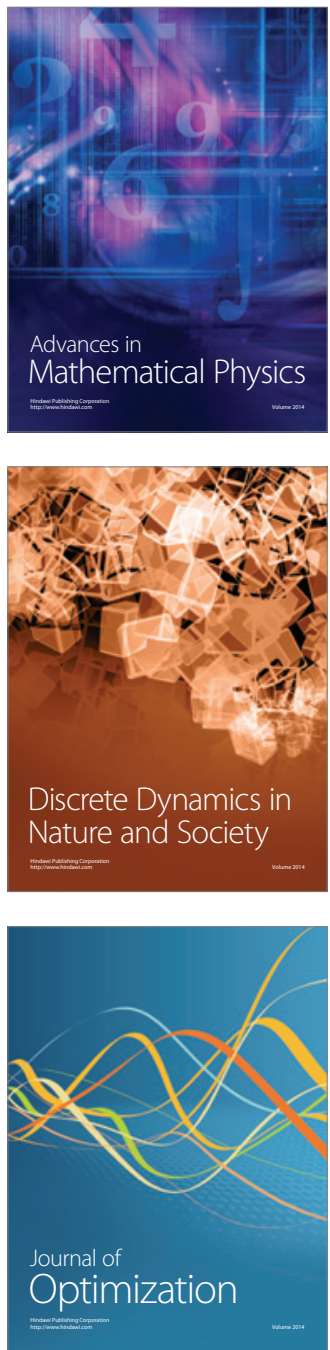